\title{
Dan L Duncan Cancer Center
}

National Cancer Institute

\section{Source}

National Cancer Institute. Dan L Duncan Cancer Center. NCI Thesaurus. Code C105622.

The Dan L Duncan Cancer Center focuses on cancer research, patient care, training, and education. It became an $\mathrm{NCl}$-designated cancer center in 2007. It is a consortium made up of Baylor College of Medicine and its three primary teaching hospital affiliates: Ben Taub Hospital, Michael E DeBakey Veterans Affairs Medical Center, and Texas Children's Hospital. 